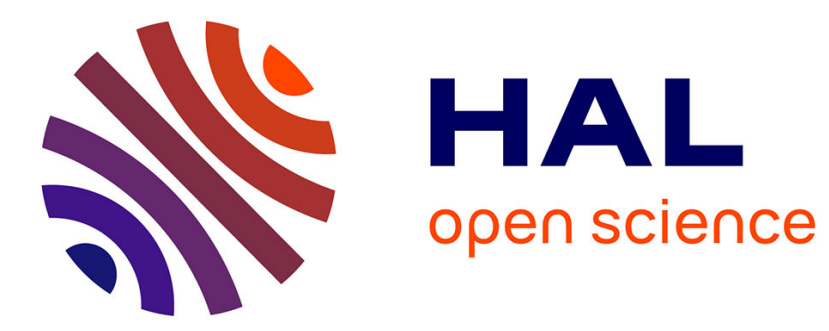

\title{
Non-resonant modes in plasmonic holey metasurfaces for the design of artificial flat lenses
}

\author{
Guido Valerio, Zvonimir Sipus, Anthony Grbic, Oscar Quevedo-Teruel
}

\section{To cite this version:}

Guido Valerio, Zvonimir Sipus, Anthony Grbic, Oscar Quevedo-Teruel. Non-resonant modes in plasmonic holey metasurfaces for the design of artificial flat lenses. Optics Letters, 2017, 42 (10), pp.20262029. 10.1364/OL.42.002026. hal-01551791

\section{HAL Id: hal-01551791 \\ https://hal.sorbonne-universite.fr/hal-01551791}

Submitted on 12 Mar 2020

HAL is a multi-disciplinary open access archive for the deposit and dissemination of scientific research documents, whether they are published or not. The documents may come from teaching and research institutions in France or abroad, or from public or private research centers.
L'archive ouverte pluridisciplinaire HAL, est destinée au dépôt et à la diffusion de documents scientifiques de niveau recherche, publiés ou non, émanant des établissements d'enseignement et de recherche français ou étrangers, des laboratoires publics ou privés. 


\title{
Non-Resonant Modes in Plasmonic Holey Metasurfaces for the Design of Artificial Flat Lenses
}

\author{
Guido VAlerio,, ${ }^{1,}$ Zvonimir Sipus, ${ }^{2}$ Anthony Grbic, ${ }^{3}$ \\ Oscar QueVEDO-TeRUeL ${ }^{4}$
}

\author{
${ }^{1}$ Sorbonne Universités, UPMC Univ Paris 06, UR2, L2E, 75252 Paris, France \\ ${ }^{2}$ Faculty of Electrical Engineering and Computing, University of Zagreb, 10000 Zagreb, Croatia, \\ ${ }^{3}$ Department of Electrical Engineering and Computer Science, The University of Michigan, 48109-2122 Ann Arbor, MI, USA, \\ ${ }^{4}$ Department of Electromagnetic Engineering, KTH Royal Institute of Technology, SE-100 44 Stockholm, Sweden \\ *Corresponding author: guido.valerio@upmc.fr
}

Received 25 January 2017; revised XX Month, XXXX; accepted XX Month XXXX; posted XX Month XXXX (Doc. ID XXXXX); published XX Month XXXX

This paper discusses non-resonant modes excited on holey metasurfaces, and their influence on the properties of spoof plasmonic states supported by the metasurface when a second surface is placed in its proximity. We consider here a metallic surface with periodic holes drilled in it. The field excited on each hole is projected onto a set of non-resonant modes in order to discuss their relative relevance. While previous simpler models were assuming only the presence of the fundamental mode, we show that the simultaneous presence of several modes occurs when the surface is placed next to a metallic plate. Therefore, higher-order modes are responsible for the peculiar physical properties of wave propagation of spoof plasmons between two surfaces, which can lead to new gradient-index flat lenses for transceivers for space communications.

OCIS codes: (240.6680) Surface plasmons; (160.3918) Metamaterials; (350.5500) Propagation.

http://dx.doi.org/10.1364/OL.99.099999

Over the past years, propagation of plasmon waves [1]-[3] along perfect conductors (PEC) with structured geometry has been explored. Holes or corrugations drilled into a PEC surface have been shown to support surface waves, named "spoof plasmons," resembling those supported by a plasmonic surface [4]-[12]. They are of great interest from a theoretical and practical perspective [13]. In several areas micro-structured surfaces are gaining ground [14]-[18], due to their simple realization, low profile and the ability to make them conformal [19]. Recently, the use of two drilled metasurfaces in a glide-symmetric arrangement was shown to completely suppress frequency dispersion, thus obtaining a constant effective refractive index over an ultra-wide frequency bandwidth [20].
In order to explain the propagation of spoof plasmons, several equivalent-plasma models have been proposed in the past [6]-[9]. However, no accurate models have been discussed for holey surfaces strongly interacting with a ground plane. For example, recent research shows that placing a conductor atop a holey surface gives rise to interesting phenomena that cannot be predicted by existing models [20], [21].

In view of these developments, we propose a simple but accurate model of a holey metallic surface with a top conducting plate (Fig. 1 ), and discuss the impact of high-order harmonics and multiple non-resonant modes on the computation of the eigenstates of the electric and magnetic fields. A set of modes is used to express the field on each hole. The non-resonant nature of each mode is due to the small size of each hole, where the mode is defined. The modal amplitudes are found by enforcing field continuity between the inplate region and the drilled regions. While in previous simpler models only the fundamental mode was retained [6], we show that a certain number of them should be considered in order to correctly predict the dispersive properties of the structure. The impact of these modes on the equivalent refractive index seen by the spoof plasmon wave is then discussed.

The formulation introduced in this paper, does not only explain the complexity of the spoof plasmonics waves, but can also be used to rapidly solve for the propagation characteristics of these structures, which could find broad application in space technology. The structure considered in this letter is shown in Fig. 1. A perfect electric conductor (PEC) is drilled with a periodic array of vertical holes with square cross section, and dimensions $a \times a$. The spatial period is $d$. On top of the holey surface, another PEC (not drilled) is placed along the $z=-t$ plane. The holes are closed on the bottom side at $z=h$ by a PEC. Note that arbitrarily shaped holes could also be chosen and the same qualitative phenomena would appear. The time dependency of all fields is assumed to be $e^{-i \omega t}$ and suppressed in the formulation. 


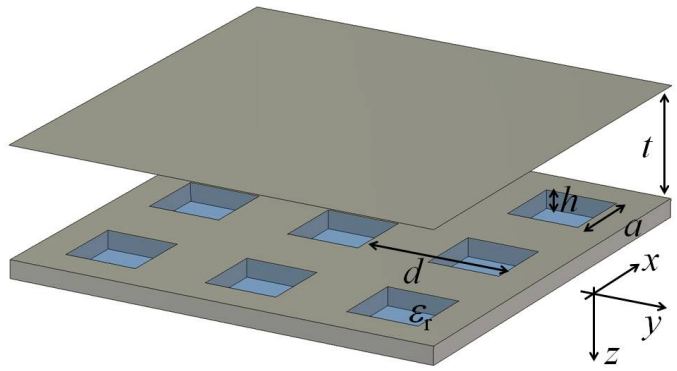

Fig. 13 -D view of the structure studied in this paper: holey perfect conductor placed at $z=0$ with a perfect conductor on the top $z=-t$. The holes have a squared cross section of lateral dimension $a$ and are in a periodic squared lattice of spatial period $d$. The depth of the holes is $\mathrm{h}$, and they are terminated with PEC. Each hole is filled with a dielectric material of refractive index $n$.

The structure can be regarded as the junction between different domains. A parallel-plate waveguide (PPW) is delimited by the two PEC plates at $z=-t$ and $z=0$. The bottom plate has a periodic array of rectangular waveguides (i.e. rectangular holes), which are closed with a PEC at $z=h$. These holes may be filled with a dielectric material $\left(\varepsilon_{\mathrm{r}}\right)$.

As in a previous model [6], the fields in the two regions can easily be expressed. Above the holey surface at $z=0^{-}$, the horizontal electric field is

$$
\left.\left|\mathbf{E}_{\mathrm{t}}\right|_{z=0^{-}}\right\rangle=\frac{1}{d^{2}} \sum_{p q} \sin \left(k_{z, p q} t\right)\left(\begin{array}{c}
A_{p q}^{x} \\
A_{p q}^{y}
\end{array}\right)\left|e^{i\left(k_{x, p} x+k_{y, q} y\right)}\right\rangle
$$

where a sum of spatial harmonics $(p, q)$ has been used by virtue of the periodicity. $A_{p q}^{x}$ and $A_{p q}^{y}$ are the amplitudes of each spatial harmonic, $k_{x, p}$ and $k_{y, q}$ are the wavenumbers describing the $x y$ propagation of the $(p, q)$ th harmonic, with $k_{x, p}=k_{x}+2 \pi p / d$ and $k_{y, p}=k_{y}+2 \pi q / d$, and the corresponding vertical wavenumber $k_{z, p q}=\sqrt{k_{0}^{2}-k_{x, p}^{2}-k_{y, q}^{2}}$ given by the separation relation.

Accordingly, the horizontal magnetic field at $z=0^{-}$can be derived leading to the following expression:

$$
\begin{aligned}
& \left.\left|\eta_{0} \mathbf{H}_{\mathrm{t}}\right|_{z=0^{-}}\right\rangle=\frac{1}{i k_{0} d^{2}} \sum_{p q} \cos \left(k_{z, p q} h_{\mathrm{PPW}}\right) \\
& \quad \times\left(\begin{array}{c}
-A_{p q}^{x} \frac{k_{x, p} k_{y, q}}{k_{z, p q}}-A_{p q}^{y} \frac{k_{0}^{2}-k_{x, p}^{2}}{k_{z, p q}} \\
A_{p q}^{x} \frac{k_{0}^{2}-k_{y, q}^{2}}{k_{z, p q}}+A_{p q}^{y} \frac{k_{x, p} k_{y, q}}{k_{z, p q}}
\end{array}\right)\left|e^{i\left(k_{x, p} x+k_{y, q} y\right)}\right\rangle
\end{aligned}
$$

The horizontal electric and magnetic fields inside the rectangular waveguide at $z=0^{+}, 0 \leq x, y \leq a$ are

$$
\begin{gathered}
\left.\left|\mathbf{E}_{\mathrm{t}}\right|_{z=0^{+}}\right\rangle=\sum_{(m, n)} r_{m n}^{-} C_{m n}^{h}\left|\boldsymbol{\Phi}_{m n}^{h}(x, y)\right\rangle+r_{m n}^{-} C_{m n}^{e}\left|\boldsymbol{\Phi}_{m n}^{e}(x, y)\right\rangle \\
\left.\left|\eta_{0} \mathbf{H}_{\mathrm{t}}\right|_{z=0^{+}}\right\rangle=\sum_{(m, n)} C_{m n}^{h} r_{m n}^{+} \frac{q_{m n}}{k_{0}}\left|\hat{\mathbf{z}} \times \boldsymbol{\Phi}_{m n}^{h}(x, y)\right\rangle \\
+C_{m n}^{e} r_{m n}^{+} \frac{k_{0} \varepsilon_{\mathrm{r}}}{q_{m n}}\left|\hat{\mathbf{z}} \times \boldsymbol{\Phi}_{m n}^{e}(x, y)\right\rangle
\end{gathered}
$$

The fields have been expressed as a sum over the $(m, n)$ th $\mathrm{TE}^{\mathrm{z}}$ (subscript ' $h$ ') and $\mathrm{TM}^{\mathrm{z}}$ (subscript ' $\mathrm{e}$ ') modes travelling inside the hole along the vertical $z$ direction [22]:

$$
\begin{array}{r}
\left|\boldsymbol{\Phi}_{m n}^{h}(x, y)\right\rangle=\left|\hat{\mathbf{x}} n \varphi_{m n}^{x}(x, y)-\hat{\mathbf{y}} m \varphi_{m n}^{y}(x, y)\right\rangle, \\
\left|\boldsymbol{\Phi}_{m n}^{e}(x, y)\right\rangle=\left|\hat{\mathbf{x}} m \varphi_{m n}^{x}(x, y)+\hat{\mathbf{y}} n \varphi_{m n}^{y}(x, y)\right\rangle
\end{array}
$$

where the orthonormal scalar functions are

$$
\varphi_{m n}^{x}(x, y)=\left\{\begin{array}{cl}
\frac{2}{a} \cos \left(\frac{m \pi x}{a}\right) \sin \left(\frac{n \pi y}{a}\right) & m \neq 0 \\
\frac{\sqrt{2}}{a} \sin \left(\frac{n \pi y}{a}\right) & m=0
\end{array}\right.
$$

and $\varphi_{m n}^{y}(x, y)=\varphi_{n m}^{x}(y, x)$. The coefficients $C_{m n}^{e / h}$ are the amplitudes of the above-mentioned hole modes. The reflection coefficients are $r_{m n}^{-}=1-e^{2 i q_{m n} h}, r_{m n}^{+}=1+e^{2 i q_{m n} h}$, and the $(m, n)$ th wavenumber is $q_{m n}=\sqrt{k_{0}^{2} \varepsilon_{r}-\left(m^{2}+n^{2}\right) \pi^{2} / a^{2}}$ inside the hole.

By enforcing the continuity of the electric field on the whole unit cell in $[0, d] \times[0, d]$ at $z=0$, and projecting this condition on each spatial harmonic $\left\langle e^{i\left(k_{x p} x+k_{y q} y\right)}\right|$ we obtain the harmonic coefficients $A_{p q}^{x}, A_{p q}^{y}$ as a function of the hole modal amplitudes $C_{i .}$. Once placed into the magnetic field expression (2), $\left.\eta_{0} \mathbf{H}_{\mathrm{t}}\right|_{z=0^{-}}$is function of only the amplitudes $C_{i}$. The continuity of the magnetic field across the hole aperture can now be enforced and projected on the set of modes $\left\langle\varphi_{m^{\prime} n^{\prime}}^{x}(x, y)\right|$ and $\left\langle\varphi_{m^{\prime} n^{\prime}}^{y}(x, y)\right|$. As a final result, a homogeneous linear system is obtained,

$$
\underline{\mathbf{M}} \cdot \mathbf{C}=\underline{\mathbf{M}} \cdot\left(\begin{array}{c}
C_{01}^{h} \\
\vdots \\
C_{11}^{h} \\
C_{11}^{e} \\
\vdots
\end{array}\right)=0
$$

The entries of the matrix $\underline{\mathbf{M}}$ are of the form (8), where $\tilde{\varphi}_{m^{\prime} n^{\prime}}^{x / y}=\left\langle\varphi_{m^{\prime} n^{\prime}}^{x / y} \mid e^{i\left(k_{x p} x+k_{y q} y\right)}\right\rangle$, and $\tilde{\boldsymbol{\Phi}}_{m n}^{h / e}=\left\langle e^{i\left(k_{x p} x+k_{y q} y\right)} \mid \boldsymbol{\Phi}_{m n}^{h / e}\right\rangle$. The first (second) line in (8) is used for the lines of $\underline{\mathbf{M}}$ coming from projections of the $\mathbf{H}_{\mathrm{t}}$ continuity on the $x(y)$ components of each mode. Entries with 'e' ('h') superscript multiply modes with the same polarization in (7). The plus (minus) sign in the second line of (8) is used for $\mathrm{h}(\mathrm{e})$ modes. $f$ and $g$ are functions of the frequency and parameters of the groves only. Their expressions are not given here for the sake of brevity.

The wavenumbers $k_{x}$ of the eigenstate of the system are determined by searching for the zeros of the determinant of $\underline{\mathbf{M}}$, at each angular frequency $\omega$. The function $k_{x}(\omega)$ is thus used to study the frequency dispersion of the spoof plasmon supported by the holey PPW. The eigenstates $\mathbf{C}$ can equally be found as a function of the frequency, and the amplitudes of each mode can be evaluated. In the following results, the normalization $\mathbf{C} \cdot \mathbf{C}^{*}=1$ is assumed.

We use the semi-analytical formulation described previously to obtain and discuss the dispersion characteristics of the holey PPW in Fig. 1, for different distances between the plates. The zeroes of the determinant of $\underline{\mathbf{M}}$ are found by using the Padé-approximantbased root-finding procedure approach reported in [23]. The modal expansion is compared with a periodic finite-element method (FEM) commercial solver; CST Microwave Studio. The analyses are done for simplicity for $k_{y}=0$ (Floquet-mode propagating along the $x$ direction), but the formulation is valid for a 


$$
M_{m n}^{m^{\prime} n^{\prime}}=\left\{\begin{array}{l}
\sum_{p, q} \frac{-r_{m n}^{-}}{\tan \left(k_{z, p q} t\right)}\left[\frac{k_{0}^{2}-k_{x, p}^{2}}{i d^{2} k_{z, p q}} \hat{\mathbf{y}} \cdot \tilde{\boldsymbol{\Phi}}_{m n}^{h / e}\left(k_{x, p}, k_{y, q}\right) \tilde{\varphi}_{y}^{m^{\prime} n^{\prime}}\left(k_{x, p}, k_{y, q}\right)+\frac{k_{x, p} k_{y, q}}{i d^{2} k_{z, p q}} \hat{\mathbf{x}} \cdot \tilde{\boldsymbol{\Phi}}_{m n}^{h / e}\left(k_{x, p}, k_{y, q}\right) \tilde{\varphi}_{y}^{m^{\prime} n^{\prime}}\left(k_{x, p}, k_{y, q}\right)\right]+f_{m n}(\omega) \\
\sum_{p, q} \frac{ \pm r_{m n}^{-}}{\tan \left(k_{z, p q} t\right)}\left[\frac{k_{0}^{2}-k_{y, q}^{2}}{i d^{2} k_{z, p q}} \hat{\mathbf{x}} \cdot \tilde{\boldsymbol{\Phi}}_{m n}^{h / e}\left(k_{x, p}, k_{y, q}\right) \tilde{\varphi}_{x}^{m^{\prime \prime} n^{\prime}}\left(k_{x, p}, k_{y, q}\right)+\frac{k_{x, p} k_{y, q}}{i d^{2} k_{z, p q}} \hat{\mathbf{y}} \cdot \tilde{\boldsymbol{\Phi}}_{m n}^{h / e}\left(k_{x, p}, k_{y, q}\right) \tilde{\varphi}_{x}^{m^{\prime} n^{\prime}}\left(k_{x, p}, k_{y, q}\right)\right]+g_{m n}(\omega)
\end{array}\right.
$$

skew propagation axis. The horizontal axis shows $\beta=\mathfrak{R}\left\{k_{x}\right\}$ of the surface plasmon wave. The harmonic series in the matrix entries (8) are truncated by retaining the terms $p=0, \pm 1, \ldots, \pm N_{f}$, $q=0, \pm 1, \ldots, \pm N_{f}$, thus keeping a total number of $\left(2 N_{f}+1\right)^{2}$ terms.

First, we will focus on the interaction of the holes through higherorder spatial harmonics. In Fig. 2(a) (a "thick" PPW, $t=2 \mathrm{~mm}$ ), result when only the $(0,0)$ harmonic is retained, as suggested in 6 , are compared with those obtained with the $N_{\mathrm{f}}=1$ (dotted green line), which perfectly agree with FEM results (blue squares). In this case, a single $\mathrm{TE}_{01}$ mode has been retained in the formulation, leading to accurate results. In Fig. 2(b) (a "thin" PPW, $t=0.1 \mathrm{~mm}$ ), results with $N_{\mathrm{f}}=1$ and one mode (dotted green line) are rather far from the accurate FEM results, while the curve with $N_{\mathrm{f}}=3$ (dashed green line) and one mode on the hole is closer to the accurate result (blue squares).

The importance of higher-order harmonics can be explained by noticing that electric field can have a non-negligible spatial variation along the $x$ and $y$ axes (being different from constant along the hole, and zero outside). A spatial Fourier series needs a certain number of harmonics to follow these variations, and cannot be reduced to the $(0,0)$ th term only.

Alternatively, the formulation of the previous section can shed additional light on the importance of higher-order harmonics. When computing the series in (8), the factors $\frac{k_{0}^{2}-k_{x, p}^{2}}{k_{z, p q}}, \frac{k_{0}^{2}-k_{y, q}^{2}}{k_{z, p q}}$ and $\frac{k_{x, p} k_{y, q}}{k_{z, p q}}$ grow with the $(p, q)$ indexes. Convergence is granted of course by the spectral modal functions $\tilde{\boldsymbol{\Phi}}_{m n}^{h / e}\left(k_{x, p}, k_{y, q}\right) \tilde{\varphi}^{m^{\prime} n^{\prime}}\left(k_{x, p}, k_{y, q}\right)$. However, in the first few terms of the sums this decay does not occur yet, and various terms need to be considered as well as the fundamental one. This is in contrast to the simpler 2D corrugated surfaces reported in [6].

Another important physical phenomenon is the excitation of different modes in the holes as the geometrical parameters of the structure changes. In the thick case ( $t=2 \mathrm{~mm}$ in Fig. 2(a)), one single mode on the hole leads to a very good agreement with the FEM results. On the other hand, in the more extreme case of Fig. 2(b) $(t=0.1 \mathrm{~mm})$, the relevance of multiple modes on the hole is more evident. The green curves are obtained by retaining one mode only, the TE01. Both curves differ appreciably from the finiteelement results shown with blue squares. However, very good agreement is obtained for the red curve, where we have retained the modes $\mathrm{TE}_{m n}$ and $\mathrm{TM}_{m n}$ with $m=0,1(M=\max (m)=1)$ and $n=$ $1,3,5(N=\max (n)=5)$ (since the structure is symmetric along $y$ and $k_{y}=0$, only odd $n$ contribute to the total field in this specific case).
This confirms the results reported in [9], where a multimode approach was used for an open holey metasurface. Note that the number of modes in the waveguide, $N$ and $M$, is not directly related to the number of PPW modes, $N_{f}$. In fact, the number of required waveguide modes depends on the hole size, while the number of PPW modes depends on the length of the period.

The need for a higher number of waveguide modes is due to the presence of the plate, which enforces a vertical electric field in the proximity of the hole, corresponding to TM modes inside.

As a result, we can state that variation in distance between the upper plate and the holey surface can enhance or suppress higherorder modes within the hole. Correspondingly, this modifies the dispersive diagram shown in the pictures, namely the values of $\beta / k_{0}$. This quantity can be considered as an effective refractive index seen by the plasmonic wave travelling along the surface. The possibility of modifying the refractive index without changing the materials used is very attractive for the design of flat artificial graded-index lenses [19]. It is also of great interest to microwave component and antenna design for space applications, where dielectric materials are avoided.

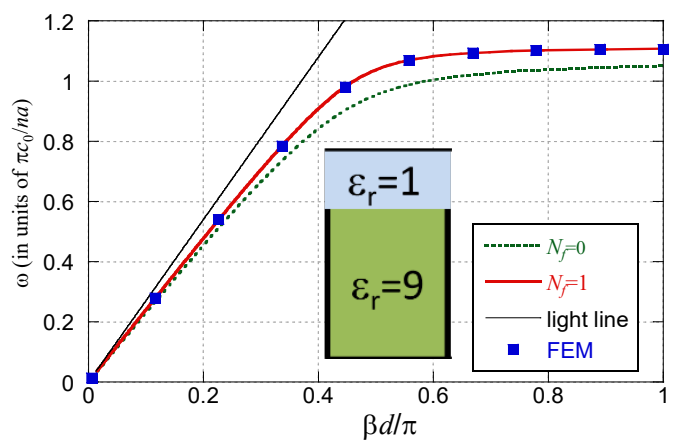

(a)

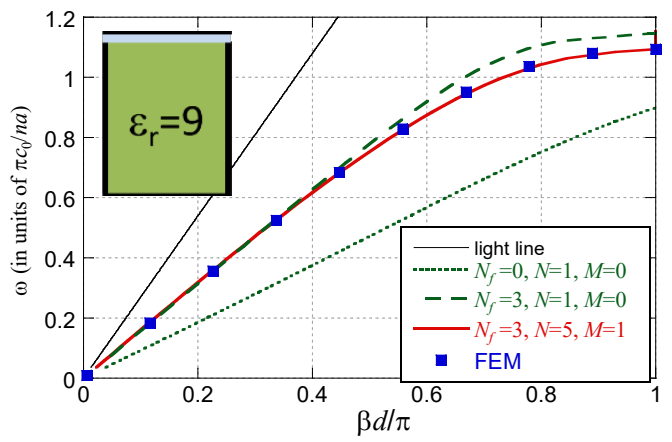

(b)

Fig. 2 Dispersion analysis of a holey PEC with a PEC plate on the top at distance $h$. The plasmonic wave propagates in the air on top of the hole (in blue in the color picture). Geometric and physical parameters: $a / d=$ $0.9, n=3, h=5 \mathrm{~mm}, d=4 \mathrm{~mm}$. (a) $t=2 \mathrm{~mm}$, (b) $t=0.1 \mathrm{~mm}$. 
In order to further explore the presence of different hole modes, we compute the eigenstate $\mathbf{C}$ from (7) for the two geometries analyzed in Fig. 2. The set of modes $m=0,1$ and $n=1,3,5$ (for a total of 9 modes) has been used, since previous results prove it to give an accurate description of the frequency dispersion. In both cases, the coefficients of $\mathbf{C}$ with the largest magnitude correspond to the $\mathrm{TE}_{01}$ and $\mathrm{TM}_{11}$ modes. The others are smaller than 0.2 over the entire frequency range. The magnitudes of these two largest components are shown in Fig. 3. Retaining only the fundamental mode $\mathrm{TE}_{01}$ does not accurately describe the field at frequencies where $\mathrm{TM}_{11}$ is dominant. In the insets, the total fields are shown for three frequencies $(2,8$ and $12 \mathrm{GHz})$ as the sum of the 9 modes retained in the modal expansion. Different field distribution on the hole aperture are evident as the frequency and the distance between plates changes.

A simple change in the geometrical parameters can be used to modify the effective refractive index of the plasmon to realize lowcost and low-profile graded-index flat lenses. In Fig. 4, the equivalent refractive index is calculated for different hole depths $h$ : a parameter that is simple to modify in fabrication. A method for synthesizing non-dispersive refractive indexes has been recently proposed by facing two holey surfaces, translated one half a period with respect to each other (a glide-symmetric structure) [20;21]. The modes in this more complex structure can be analyzed with a formulation very similar to the one reported here, and will be the object of a future study.

In conclusion, the impact of multiple non-resonant modes has been studied on the equivalent refractive index of a 2D-periodic holey surface backed by a metallic ground plane, and coupling amongst cells through higher-order harmonics. The PPW structure can be used to design fully metallic, planar gradient-index lenses for space microwave and THz transmitters and receivers [14].

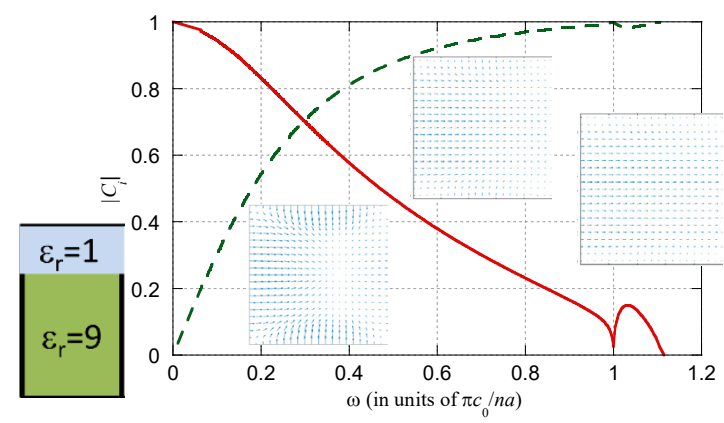

(a)

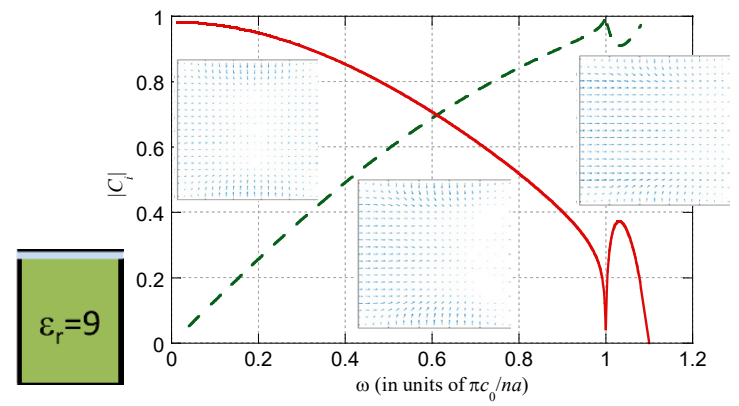

(b)

Fig. 3 Magnitude of the largest components of $\mathbf{C}$ : the $\mathrm{TE}_{01}$ mode (green dashed line) and the TM11 mode (red solid line). Parameters: $a / d=0.9$, $n=3, h=5 \mathrm{~mm}, d=4 \mathrm{~mm}$. (a) $t=2 \mathrm{~mm}$, (b) $t=0.1 \mathrm{~mm}$.

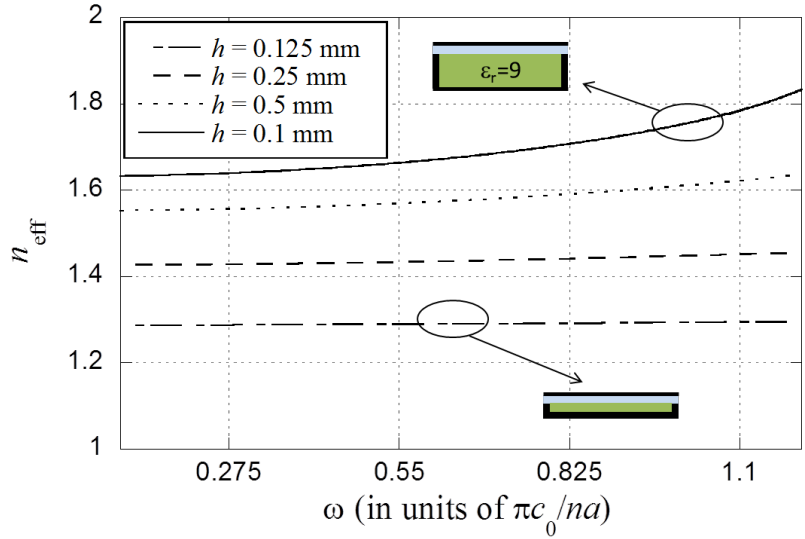

Fig. 4 Equivalent refractive index as a function of frequency of the structure in Fig. 1 with $t=0.1 \mathrm{~mm}, p=4 \mathrm{~mm}, a / d=0.9, n=3$.

Funding. This work was supported by the Spanish Government Project TEC2013-44019-R, by the Swedish STINT Postdoctoral Transition Grant PT2014-5813 (by the Swedish Foundation for International Cooperation in Research and Higher Education), and by the French ANR Grant HOLeYMETA ANR-16-CE24-0030.

\section{References}

1. C. H. Walter, IRE Trans Antennas Propag, 8, 508 (1960).

2. R. H. Ritchie, Phys. Rev., 106, 874 (1957).

3. W. L. Barnes, A. Dereux, T. W. Ebbesen, Nature 424, 824 (2003).

4. J. B. Pendry, L. Martín-Moreno, and F. J. Garcia-Vidal, Science 305, 847 (2004).

5. A. Grbic and G. V. Eleftheriades, Phys. Rev. Lett., 92, 117403 (2004).

6. J. B. Pendry, L. Martín-Moreno and F. J. Garcia-Vidal, J. Opt. A: Pure Appl. Opt. 7 S97 (2005)

7. A. Rusina, M. Durach, M. I. Stockman, Appl Phys A, 100, 375 (2010).

8. Z. Li, B. Xu, L. Liu, J. Xu, C. Chen, C. Gu, and Y. Zhou, Sci. Rep. 6, 21199 (2016).

9. F. J. Garcia de Abajo and J. J. Saenz, Phys. Rev. Lett., 95, 233901 (2005)

10. A. Panaretos and D. Werner, Opt. Express, 24, 2443 (2016).

11. D. Martin-Cano, O. Quevedo-Teruel, E. Moreno, L. Martin-Moreno, F. J. Garcia-Vidal., Optics Lett. 36, 4635 (2011).

12. R. Quesada, D. Martın-Cano, F. J. Garcıa-Vidal, and J. Bravo-Abad, Opt. Lett., 39, 2990 (2014).

13. M. Nesterov, D. Martin-Cano, A. Fernandez-Dominguez, E.Moreno, L. Martin-Moreno, and F. Garcia-Vidal, Opt. Lett. 35, 423 (2010).

14. R. Williams, S. R. Andrews, S. A. Maier, A. I. Fernández-Domínguez, L. Martín-Moreno, and F. J. García-Vidal, Nature Photonics 2, 175 (2008)

15. T. Zentgraff, Y. Liu, M. H. Mikkelsen, J. Valentine, and X. Zhang. Nat. Nano. 6, 151155 (2011).

16. S. Maci, G. Minatti, M. Casaletti, and M. Bosiljevac, IEEE Antenn. Wirel. Propag. Lett. 10, 1499 (2011).

17. J. A. Dockrey, M. J. Lockyear, S. J. Berry, S. A. R. Horsley, J. R. Sambles, and A. P. Hibbins, Phys. Rev. B, 87, 125137 (2013).

18. J. Renger, M. Kadic, G. Dupont, S. S. Aćimović, S. Guenneau, Romain Quidant, and Stefan Enoch, Optics Express, 18, 15757-15768 (2010)

19. R. C. Mitchell-Thomas, O. Quevedo-Teruel, T. M. McManus, S. A. R. Horsley, and Y. Hao, Optics Letters, 39, 3551 (2014).

20. O. Quevedo-Teruel, M. Ebrahimpouri, and M. Ng Mou Kehn, IEEE Antennas Wireless Prop. Lett, 15, 484 (2016).

21. G. Valerio, Z. Sipus, A. Grbic, and O. Quevedo-Teruel, in press IEEE Trans Antennas Propag., 65, (2017).

22. L. Felsen and N. Marcuvitz, Propagation and Scattering of Waves. WileyIEEE Press, 1994.

23. V. Galdi, and I. M. Pinto, Microw. Opt. Technol. Lett., 24, 135 (2000). 